\title{
Ácido linoleico na prevenção do infarto agudo do miocárdio: uma revisão integrativa
}

\author{
Linoleic acid in the prevention of acute myocardial infarction: an integrative review \\ El ácido linoleico en la prevención del infarto agudo de miocardio: una revisión integrativa
}

Gabrielle Guimarães Araújo

ORCID: https://orcid.org/0000-0003-0314-4061

Faculdade de Medicina de Olinda, Brasil

E-mail: gaby_mello_@hotmail.com

Thaís Carolline de Jesus Souza

ORCID: https://orcid.org/0000-0002-3677-5874

Faculdade de Medicina de Olinda, Brasil

E-mail: thais.carolline3@gmail.com

Nicole Silva Flor

ORCID: https://orcid.org/0000-0002-1455-400X

Faculdade de Medicina de Olinda, Brasil

E-mail: nicole.flor15@hotmail.com

Alice Joana Souza Vieira da Silva

ORCID: https://orcid.org/0000-0002-7347-0690

Faculdade de Medicina de Olinda, Brasil E-mail: alicejoana2010@hotmail.com

Thárcia Kiara Beserra de Oliveira

ORCID: https://orcid.org/0000-0001-6352-7254

Faculdade de Medicina de Olinda, Brasil

E-mail: tharcia_kiara@hotmail.com

\begin{abstract}
Resumo
Introdução: As doenças cardiovasculares, como o infarto agudo do miocárdio (IAM), são uma das principais causas de morte no Brasil e no mundo, afetando aproximadamente 183 para 100.000 habitantes. Se torna importante o controle da pressão arterial, e principalmente da dislipidemia observada na maioria dos pacientes através de dietas. Objetivos: avaliar os efeitos do ácido linoleico na prevenção do infarto agudo do miocárdio. Metodologia: Esta é uma revisão abrangente da literatura, que revisa rigorosamente e combina pesquisas com diferentes métodos. Para seleção dos estudos, foi realizada uma leitura minuciosa dos títulos e dos resumos, a fim de verificar quais se encaixavam na questão norteadora e nos critérios de inclusão e exclusão. Resultados e discussão: O ácido linoleico é um precursor do ácido araquidônico, que participa da síntese de mediadores biologicamente ativos, como prostaglandinas, tromboxanos e leucotrienos. Essas substâncias atuam como mediadores inflamatórios, estimulantes e cicatrizantes. Pacientes com infarto agudo do miocárdio (IAM) podem se beneficiar de suplementação ácidos graxos poliinsaturados. Conclusão: A ingestão dos ácidos graxos está relacionada com a diminuição do risco de paradas cardíacas, possuindo efeito regulador sobre as propriedades elétricas do miocárdio, reduzindo as arritmias ventriculares e o risco de morte súbita.
\end{abstract}

Palavras-chave: Ácido linoleico; Ácidos graxos ômega 6; Infarto agudo do miocárdio.

\begin{abstract}
Introduction: Cardiovascular diseases, such as acute myocardial infarction (AMI), are one of the main causes of death in Brazil and worldwide, affecting approximately 183 per 100,000 inhabitants. It becomes important to control blood pressure, and especially dyslipidemia observed in most patients through diets. Objectives: to evaluate the effects of linoleic acid in the prevention of acute myocardial infarction. Methodology: This is a comprehensive literature review that rigorously reviews and combines research using different methods. To select the studies, a thorough reading of the titles and abstracts was performed, in order to verify which ones fit the guiding question and the inclusion and exclusion criteria. Results and discussion: Linoleic acid is a precursor of arachidonic acid, which participates in the synthesis of biologically active mediators, such as prostaglandins, thromboxanes and leukotrienes. These substances act as inflammatory, stimulant and healing mediators. Patients with acute myocardial infarction (AMI) may benefit from polyunsaturated fatty acid supplementation. Conclusion: The intake of fatty acids is related to a decrease in the risk of cardiac arrest, having a regulatory effect on the electrical properties of the myocardium, reducing ventricular arrhythmias and the risk of sudden death.
\end{abstract}

Keywords: Linoleic acid; Omega 6 fatty acids; Acute myocardial infarction. 


\begin{abstract}
Resumen
Introducción: Las enfermedades cardiovasculares, como el infarto agudo de miocardio (IAM), son una de las principales causas de muerte en Brasil y en el mundo, afectando aproximadamente a 183 por 100.000 habitantes. Se vuelve importante controlar la presión arterial, y especialmente la dislipidemia que se observa en la mayoría de los pacientes a través de las dietas. Objetivos: evaluar los efectos del ácido linoleico en la prevención del infarto agudo de miocardio. Metodología: Se trata de una revisión exhaustiva de la literatura que revisa y combina rigurosamente la investigación utilizando diferentes métodos. Para la selección de los estudios se realizó una lectura minuciosa de los títulos y resúmenes, con el fin de verificar cuáles se ajustaban a la pregunta orientadora y los criterios de inclusión y exclusión. Resultados y discusión: El ácido linoleico es un precursor del ácido araquidónico, que participa en la síntesis de mediadores biológicamente activos, como prostaglandinas, tromboxanos y leucotrienos. Estas sustancias actúan como mediadores inflamatorios, estimulantes y curativos. Los pacientes con infarto agudo de miocardio (IAM) pueden beneficiarse de la suplementación con ácidos grasos poliinsaturados. Conclusión: La ingesta de ácidos grasos se relaciona con una disminución del riesgo de paro cardíaco, teniendo un efecto regulador sobre las propiedades eléctricas del miocardio, reduciendo las arritmias ventriculares y el riesgo de muerte súbita.
\end{abstract}

Palabras clave: Ácido linoleico; Ácidos grasos omega 6; Infarto agudo del miocardio.

\title{
1. Introdução
}

As doenças cardiovasculares, como o infarto agudo do miocárdio (IAM), são uma das principais causas de morte no Brasil e no mundo, afetando aproximadamente 183 para 100.000 habitantes, sendo mais prevalente no sexo masculino, 1,7 vezes, do que no sexo feminino. A principal causa é a aterosclerose, e seus fatores de risco estão relacionados ao tabagismo, sedentarismo, consumo excessivo de carnes vermelhas, leite, gorduras e alimentos processados, sobrepeso e hipertensão. O controle dos fatores de risco do infarto agudo do miocárdio, tem sido a base para a prevenção. Nesse sentido, se torna importante o controle da pressão arterial, e principalmente da dislipidemia observada na maioria dos pacientes através de dietas que reduzam os níveis de triglicerídeos e elevem os níveis de HDL-c (Santos et al., 2013; Mertins et al., 2016; Santos et al., 2018).

Até o início do século XX, era conceituado de que ácidos graxos dispunham propriedades de armazenar energia, podendo ser sintetizados pelo organismo desde os carboidratos até proteínas. Portanto, o ácido linoleico é um composto natural que existe em diferentes formas de acordo com o arranjo de suas moléculas. Desse modo, caracterizam os isômeros geométricos do ácido linoleico (C18: 2), que contêm duas ligações duplas e são encontrados em vários alimentos de origem animal; carne, leite, feijão, grãos e óleos vegetais (Ferraz \& Carneiro, 2017).

Existem muitos benefícios do Ácido Linoléico Conjugado (CLA) relacionado as doenças cardiovasculares, visto que participam no processo da circulação sanguínea, promovendo ações benéficas ao sistema cardiovascular. A ingestão dos ácidos graxos está relacionada com a diminuição do risco de paradas cardíacas, possuindo efeito regulador sobre as propriedades elétricas do miocárdio, reduzindo as arritmias ventriculares e o risco de morte súbita. Vale ressaltar que a maior parte do ácido linoleico é detectado na dieta alimentar, sendo assim é válida a importância do consumo do CLA seus derivados (Novello, Franceschini \& Quintiliano, 2015).

Diante do exposto, o presente estudo justifica-se pela alta prevalência de doenças cardiovasculares, em especial o IAM, que possuem como fatores de risco a obesidade e a dislipidemias, e pelas repercussões destas desordens na redução da qualidade de vida dos indivíduos acometidos, observando a necessidade de um estudo aprofundado do ácido linoleico nas possíveis alternativas para o controle dessas patologias. Com isso, o trabalho tem como objetivo avaliar os efeitos do ácido linoleico na prevenção do infarto agudo do miocárdio.

\section{Metodologia}

Esta é uma revisão integrativa, que revisa rigorosamente e combina pesquisas com diferentes métodos, e compara a incidência de doenças entre as populações de acordo com as diretrizes em evidências científicas. A pergunta que direcionou a 
revisão foi: Quais os efeitos do ácido linoleico na prevenção do infarto agudo do miocárdio?

\section{Estratégia de busca}

A busca pelos artigos foi realizada em junho de 2021 nas seguintes bases de dados: Scielo e Pubmed, utilizando os seguintes unitermos: "ácido linoleico", "ácidos graxos ômega 6" e "infarto agudo do miocárdio". A busca integrada foi realizada unindo os descritores com o operador booleano "AND".

Para seleção dos estudos, foi realizada uma leitura minuciosa dos títulos e dos resumos, a fim de verificar quais se encaixavam na questão norteadora e nos critérios de inclusão e exclusão. Após esta fase, dois revisores independentes executaram uma leitura previa de todos os artigos selecionados.

A pesquisa obedeceu aos seguintes critérios de inclusão: artigos disponíveis em texto completo de forma gratuita, publicados nos últimos dez anos, nos idiomas português, inglês e espanhol. Foram selecionados estudos que abordavam a temática específica sobre os efeitos do ácido linoleico e sua relação com a prevenção do infarto agudo do miocárdio. A lista de referências dos artigos também foi escrutinada, a fim de se identificar estudos potencialmente elegíveis. Foram desconsiderados os textos que não abordaram a questão norteadora da pesquisa ou apresentaram duplicatas em mais de uma base de dados. Os resultados são apresentados de forma sucinta e de forma integrada, após análise criteriosa, com ênfase nos principais aspectos de cada estudo.

\section{Resultados e Discussão}

A quantidade de estudos encontrados combinando os descritores citados anteriormente totalizou 162 artigos. Após essa pesquisa, os critérios de inclusão e exclusão seguindo os critérios: estudos encontrados a partir dos descritores; estudos em português ou inglês; estudos que permaneceram após aplicação dos filtros de intervalo dos últimos anos (2015-2021); estudos excluídos por se repetirem em diferentes bases ou idioma; estudos excluídos por não corresponderem a temática proposta. Chegando a amostragem final desta revisão integrativa, a qual totalizou 10 produções científicas.

As 10 produções científicas escolhidas foram descritas (Quadro 1), seguindo Título, Método e Conclusão de modo que foram resumidas destacando os principais achados.

As doenças cardiovasculares (DCV) são as principais causas de morte na população brasileira, dentre elas, destaca-se a doença arterial coronariana, que tem como efetor o infarto do miocárdio, o mesmo, resulta no agravante de que a mortalidade devido à isquemia miocárdica tem ocorrido em idades progressivamente mais precoces (Mansur et al., 2016; Luz, Santos e Sabino, 2017).

O ácido linoleico é um precursor do ácido araquidônico, que participa da síntese de mediadores biologicamente ativos, como prostaglandinas, tromboxanos e leucotrienos. Essas substâncias atuam como mediadores inflamatórios, estimulantes e cicatrizantes (Marklund et al., 2019). 
Tabela 1.

\begin{tabular}{|c|c|c|c|}
\hline $\mathbf{N}^{\mathbf{o}}$ & Título & Método & Conclusão \\
\hline 1 & $\begin{array}{l}\text { The ratio of serum } n-3 \text { to } n-6 \text { polyunsaturated fatty } \\
\text { acids is associated with diabetes mellitus in patients } \\
\text { with prior myocardial infarction: a multicenter } \\
\text { cross-sectional study. }\end{array}$ & $\begin{array}{l}\text { Estudo } \\
\text { multicêntrico } \\
\text { observacional }\end{array}$ & $\begin{array}{l}\text { Demostrou que pacientes com infarto agudo do } \\
\text { miocárdio e com diabetes, mas não com } \\
\text { hipertensão ou dislipidemia, têm menores razões } \\
\text { EPA / AA e DHA / AA. Essa associação sugere } \\
\text { que EPA / AA e DHA / AA se comportam de } \\
\text { maneira diferente em indivíduos com diferentes } \\
\text { níveis de PCR. }\end{array}$ \\
\hline 3 & $\begin{array}{l}\text { Efeitos dos ácidos graxos ômega-3, ômega-6 e } \\
\text { ômega-9 sobre o risco cardiovascular de indivíduos } \\
\text { adultos: estudo clínico de prevenção primária. }\end{array}$ & $\begin{array}{l}\text { Estudo clínico, } \\
\text { randomizado, } \\
\text { duplo-cego }\end{array}$ & $\begin{array}{l}\text { O ômega-3 possui efeito hipotrigliceridêmico e } \\
\text { mostra associação cardioprotetora do HDL em } \\
\text { indivíduos com menor conteúdo plasmático basal } \\
\text { de EPA e DHA. }\end{array}$ \\
\hline 4 & $\begin{array}{l}\text { Ingestão dietética de ácidos graxos n-3 e o risco de } \\
\text { Infarto Agudo do Miocárdio: Um estudo de caso- } \\
\text { controle, }\end{array}$ & $\begin{array}{l}\text { Estudo caso- } \\
\text { controle de base } \\
\text { hospitalar }\end{array}$ & $\begin{array}{l}\text { Este estudo revela um efeito protetor do n-3 } \\
\text { PUFA e do consumo de peixe contra o } \\
\text { AMI. Nossos resultados sugerem que existe um } \\
\text { limiar na relação quantitativa dose-resposta entre } \\
\text { a ingestão de peixes e IAM. }\end{array}$ \\
\hline 5 & $\begin{array}{l}\text { Os níveis de ácidos graxos poliinsaturados ômega-6 } \\
\text { e ômega-3 são reduzidos no sangue total de } \\
\text { pacientes italianos com um infarto do miocárdio } \\
\text { recente: o estudo AGE-IM }\end{array}$ & $\begin{array}{c}\text { Caso- controle: } \\
\text { AGE-IM }\end{array}$ & $\begin{array}{l}\text { Os níveis percentuais de PUFAs totais, PUFAs } \\
\text { ômega-3 totais e PUFAs ômega-6 totais são mais } \\
\text { baixos em pacientes com MI do que em } \\
\text { indivíduos de controle pareados na coorte AGE- } \\
\text { IM. Esses dados apoiam uma associação } \\
\text { favorável não apenas dos níveis percentuais de } \\
\text { sangue total do ômega-3 total, mas também do } \\
\text { ômega- } 6 \text { total, com o risco cardiovascular. }\end{array}$ \\
\hline 6 & $\begin{array}{l}\text { O tratamento com ácidos graxos ômega-3 pode } \\
\text { proporcionar um benefício discreto na morbilidade } \\
\text { e na mortalidade nos pacientes com insuficiência } \\
\text { cardíaca }\end{array}$ & $\begin{array}{l}\text { Ensaio clínico, } \\
\text { randomizado, } \\
\text { duplo- cego }\end{array}$ & $\begin{array}{l}\text { Um tratamento simples e seguro com ácidos } \\
\text { graxos ômega-3 pode proporcionar um benefício } \\
\text { discreto em pacientes com ICC, em términos de } \\
\text { redução de mortalidade global e morbilidade. }\end{array}$ \\
\hline 7 & Prevenção secundária em pacientes coronários & $\begin{array}{l}\text { Estudo caso- } \\
\text { controle }\end{array}$ & $\begin{array}{l}\text { Tratamentos específicos para fatores de risco } \\
\text { cardiovascular também foram desenvolvidos. } \\
\text { Portanto, o tratamento prolongado do paciente } \\
\text { coronariano evoluiu muito e o meu amigo tem um } \\
\text { controle mais completo e personalizado. }\end{array}$ \\
\hline 8 & $\begin{array}{l}\text { Associação entre a ingestão de Ácidos Graxos } \\
\text { Poliinsaturados e a menor incidência de eventos } \\
\text { cardíacos em pacientes com Diabetes Melito Tipo } 2\end{array}$ & Estudo de coorte & $\begin{array}{l}\text { Em pacientes com DM tipo 2, o maior consumo } \\
\text { de ácido graxo poliinsaturado, principalmente do } \\
\text { ácido linolênico, está associada a uma menor } \\
\text { incidência de eventos cardiovasculares. }\end{array}$ \\
\hline 9 & $\begin{array}{l}\text { Qualidade da orientação nutricional intra-hospitalar } \\
\text { em pacientes com infarto agudo do miocardio da } \\
\text { rede pública e privada de saúde em Sergipe: registro } \\
\text { VICTIM }\end{array}$ & Estudo transversal & $\begin{array}{l}\text { A orientação nutricional intra-hospitalar } \\
\text { concedida aos pacientes com IAM em Sergipe } \\
\text { possui baixa qualidade no serviço privado e } \\
\text { público, especialmente. }\end{array}$ \\
\hline 10 & $\begin{array}{l}\text { Efeito de três intervenções dietéticas sobre o perfil } \\
\text { de ácidos graxos plasmáticos em pacientes com } \\
\text { doença arterial coronariana: uma subanálise do } \\
\text { estudo GENUTRI }\end{array}$ & Subanálise & $\begin{array}{l}\text { Suplementação diária de } 30 \mathrm{ml} / \text { dia de azeite de } \\
\text { oliva extravirgem, combinado à uma dieta } \\
\text { saudável são capazes de diminuir o ácido graxo } \\
\text { palmitoleico, em } 12 \text { semanas, nos pacientes com } \\
\text { DAC. }\end{array}$ \\
\hline
\end{tabular}

Fonte: Autores (2021).

O ômega-3 possui papel cardioprotetor devido ao aumento na incorporação de EPA e DHA, o que foi reforçado em estudo clínico, randomizado, duplo-cego baseado em intervenção nutricional (3,0 g/dia de ácidos graxos) sob a fórmula de cápsulas contendo: ômega-3 (37\% de EPA e 23\% de DHA) ou ômega-6 (65\% de ácido linoleico) ou ômega-9 (72\% de ácido oleico), para comparar seus efeitos sobre os parâmetros cardiometabólicos. Observou-se também que o elevado percentual plasmático de ômega-9 se associou com partículas de LDL menos aterogênicas (fenótipo A). No que diz respeito ao ômega-6, 
os resultados mostraram que o maior tercil plasmático de ácido linoleico se associou positivamente com triglicerídeos (Pappiani, 2016).

O ácido linoleico possui possível efeito cardioprotetor, agindo sobre: o stress oxidativo, devido a redução da expressão protéica de NRF-1; aumento da expressão proteica da SERCA2a; maior capacidade de retenção ao Ca2+ para as subpopulações mitocondriais subsarcolemais (SSM) e interfibrilares (IFM) tanto na expressão dos complexos quanto da fosforilação oxidativa. Esses dados corroboram com um estudo exploratório e experimental, onde o infarto agudo do miocárdio foi induzido em ratos Wistar e analisou-se diferentes concentrações de ácido linoleico, os resultados sugerem que o tratamento com ácido linoleico na dose de $50 \mathrm{mg} / \mathrm{kg}$ por 15 dias após o infarto do miocárdio está relacionado à melhora do metabolismo mitocondrial e da contratilidade miocárdica (Rodrigues, 2017).

Os resultados obtidos acima corroboram com o estudo de Takashi et. al. (2017), que mostrou que entre os pacientes com infarto agudo do miocárdio (IAM), aqueles com diabetes melittus (DM) podem se beneficiar de suplementação ácidos graxos poliinsaturados. O principal achado foi que pacientes com IAM e com DM, mas não com hipertensão ou dislipidemia, têm menores razões de EPA e DHA, se comportando de maneira diferente em indivíduos com diferentes níveis de PCR.

A prevenção secundária em paciente coronário visa limitar o crescimento da placa de ateroma e aparecimento da síndrome coronariana aguda, que tem como o intuito limitar os sintomas e melhorar o prognostico e a sobrevida do paciente. Sendo assim, a prevenção secundária envolve o controle dos fatores de riscos nos quais se soma também ao tratamento medicamentoso, o que foi evidenciado em estudo caso-controle, realizado por Sorbets (2021).

Estudo de 171 casos incidentes ( $81 \%$ do sexo masculino < 80 anos), foram aplicados questionários indicando a frequência de consumo de alimentos previamente validados e protocolados. Resultando para n-3 PUFA levou a uma associação não significativa entre o consumo de peixes e IAM. Portanto, esse estudo demonstra a baixa relevância entre a ingestão do peixe para com o infarto agudo do miocárdio (Fernadéz, 2002).

Segundo Marangoni (2014), o estudo AGE-IM (Acidi Grassi Essenziali e Infarto Miocardico), incluindo 119 pacientes com IM e mais 103 indivíduos sem IM, nos quais realizaram exames semiológicos, como também avaliação da dieta por meio de questionário. Nesse caso, o consumo de ômega-6 e ômega-3 estão relacionados à redução do risco de infarto do miocárdio. Sendo assim, houve diminuição significativa de infarto de MI e ocorrendo o aumento dos constantes com aumento de níveis de PUFA.

Estudo clínico, randomizado, duplo-cego (Egido, 2009) demonstrou que o consumo de ômega-3 pode reduzir os riscos de mortalidade em pacientes com insuficiência cardíaca assintomática, pois incluiu-se 7.046 pacientes com ICC de causa isquêmica e não isquêmica. Concluindo que o tratamento é simples e seguro com ácidos graxos, omega- 3 proporcionam benefícios discretos em paciente com ICC, reduzindo assim a mortalidade e morbidade global em cardíacos.

Existe uma associação negativa em relação aos eventos cardíacos, aumentando o consumo de ácido linolênico, corroborando com os resultados do estudo de coorte realizado por Duarte et al. (2010), o qual submeteu os pacientes à uma avaliação nutricional de três dias com pesagem e preenchimentos de registros alimentares (RAs), acompanhados por um ano.

É sabido que uma dieta com restrição de sal, bebidas alcoólicas e aumento do consumo de cereais integrais, vegetais e hortaliças são cardioprotetores. Lima (2018) avaliou 188 voluntários, sendo mais de 80\% do serviço público em seu estudo transversal. Houve um predomínio no serviço particular de dietas restritivas em relação à sal e gorduras, cerca de $20 \%$ maior em comparação ao serviço público; os pacientes da rede particular também se beneficiaram mais no consumo de alimentos cardioprotetores.

O azeite de oliva extravirgem é fonte do ácido linoleico, contribuindo para a redução da pressão arterial sistólica, assim como do risco de doenças cardiovasculares, o que foi ressaltado por Araújo (2019) em sua subanálise, um ensaio clínico 
o qual participaram cerca de 7.440 indivíduos de ambos os sexos com alto risco para DCV que foram submetidos à intervenções dietéticas, sendo uma delas com azeite de oliva extravirgem na quantidade de 50ml/dia.

\section{Considerações Finais}

A alta prevalência de doenças cardiovasculares, em especial o Infarto Agudo do Miocárdio, impactam na qualidade de vida dos pacientes e possuem alta mortalidade. A ingestão dos ácidos graxos possui efeito regulador sobre as propriedades elétricas do miocárdio, reduzindo as arritmias ventriculares e o risco de morte súbita. O ômega-3, por sua vez, possui papel cardioprotetor devido ao aumento na incorporação de EPA e DHA. Age também sobre o stress oxidativo, devido a redução da expressão proteica de NRF-1; aumento da expressão proteica da SERCA2a; maior capacidade de retenção ao Ca2+ para as subpopulações mitocondriais subsarcolemais (SSM) e interfibrilares (IFM).

Os estudos utilizados nesta revisão integrativa mostraram a associação do aumento do consumo de ácido linolênico com a redução da pressão arterial sistólica, assim como do risco de doenças cardiovasculares. Logo, assim como uma dieta com restrição de sal, bebidas alcoólicas e aumento do consumo de cereais integrais, vegetais e hortaliças, o ácido linoleico também possui ação cardioprotetora.

Ressalta-se, então, a necessidade da execução de estratégias de prevenção do IAM sendo o ácido linoleico uma das possíveis alternativas para o controle dessas patologias, pois sua utilidade está bem fundamentada em estudos clínicos. Observamos uma maior necessidade de realizações de novos estudos em longo prazo na tentativa de trazer evidências mais robustas que sustentem o uso desse ácido graxo na prática clínica.

\section{Agradecimentos}

À Faculdade de Medicina de Olinda pelo incentivo e apoio a nossa pesquisa.

\section{Referências}

Araújo A. (2019). Efeito de três intervenções dietéticas sobre o perfil de ácidos graxos plasmáticos em pacientes com doença arterial coronariana: uma subanálise do estudo GENUTRI. Tese (Pós-graduação em Ciências da Nutrição), Universidade Federal de Ciências da Saúde de Porto Alegre.

Duarte, C. K., Santos A. L. T. Weiss T., Cantori G., Gross J. L., Azevedo M. J., \& Zelmanovitz T. (2010). Associação Entre a Ingestão de Ácidos Graxos Poliinsaturados e a Menor Incidência de Eventos Cardíacos em Pacientes com Diabetes Melito Tipo 2. Health Sciences, UFRGS.

Flores, L. E., \& Bejarano M. J. L. (2009). O tratamento com ácidos graxos ômega-3 pode proporcionar um benefício discreto na morbilidade e na mortalidade nos pacientes com insuficiência cardíaca. Rev. Science Direct. 16(7), 461.

Fernadéz E., Garrido F. A., Gutiérrez A. A., Arrigala C. F., \& Martinez-González M. A. (2002). Ingestão dietética de ácidos graxos n-3 e o risco de infarto agudo do miocárdio: um estudo de caso-controle. Rev.ScienceDirect. 118(4), 121-125.

Gonçalves N. M., \& Carneiro B. C. (2017). O ômega-3 na prevenção do infarto agudo do miocárdio. Rev. UNIANDRADE, 18(2), 86-94.

Sorbets E., Lallmahomed E., Kretz S., Cinaud A., Vallé A., Lelong H, Blachier V., \& Blacher J. (2021) Prevenção secundaria em paciente coronário. Rev. ScienceDirect. 25(2), 1-12.

Lima T. C. R. M. (2018). Qualidade da orientação nutricional intra-hospitalar em pacientes com infarto agudo do miocárdio da rede pública e privada de saúde em Sergipe: registro VICTIM. Tese (Pós-graduação em Ciências da Saúde), Universidade Federal de Sergipe.

Luz F. E., Santos B. R. M., \& Sabino W. (2017). Estudo comparativo de mortalidade por doenças cardiovasculares em São Caetano do Sul (SP), Brasil, no período de 1980 a 2010. Ciência \& Saúde Coletiva. 22(1), 161-168.

Mansur A. P., \& Favarato D. (2016). Trends in Mortality Rate from Cardiovascular Disease in Brazil, 1980-2012. Arq Bras Cardiol. 107(1), 20-25.

Marklund M., Wu J. H. U., Imamura F., Gobbo L. C., Fretts A., Goede J., Shi P., Tintle N., Wennberg M., Aslibekyan S., Chen T., Otto M. C. O., Hirakawa Y. Eriksen H. H., Kröger J., Laguzzi F., Lankinen M., Murphy R. A., Sameiri C., Virtanen J., \& Wood A. C. (2019) Biomarkers of Dietary Omega-6 Fatty Acids and Incident Cardiovascular. Disease and Mortality. American Heart Association. 139(21), 2422-2436.

Marangoni F., Novo G., Perna G., Filardi P. P., Pirelli S., Ceroti M., Querci A., \& Poli A. (2014). Os níveis de ácidos graxos poliinsaturados ômega-6 e ômega-3 são reduzidos no sangue total de pacientes italianos com um infarto do miocárdio recente: o estudo AGE-IM. Rev.ScienceDirect., 232(2), 334-338. 
Research, Society and Development, v. 10, n. 14, e390101422260, 2021

(CC BY 4.0) | ISSN 2525-3409 | DOI: http://dx.doi.org/10.33448/rsd-v10i14.22260

Mertins S. M., Kolankiewicz A. C. B., SCHMIDT C. L., Loro M. M., Poli G., Winkelmann E. R., \& Minello J. P. (2016). Prevalência de fatores de risco em pacientes com infarto agudo do miocárdio. Av. enferm. 34(1), 30-38.

Novello D., Franceschini P., \& Quintiliano D. A. (2015). A importância dos ácidos graxos $\omega-3$ e $\omega-6$ para a prevenção de doenças e na saúde humana. Rev. Salus. 2(1), 77-87.

Pappiani C. (2016). Efeitos dos ácidos graxos ômega-3, ômega-6 e ômega-9 sobre o risco cardiovascular de indivíduos adultos: estudo clínico de prevenção primária. Tese (Doutorado em Nutrição em Saúde Pública), Universidade de São Paulo, São Paulo.

Preuss M. B., Rohlfes A. L. B., Baccar N. M., Marquard L., Oliveira M. S. R., \& Shneider R. C. S. (2013). Ácido linoleico conjugado: uma breve revisão. Rev. Jovens Pesquisadores. 3(2), 134-146.

Rodrigues P. Efeitos benéficos do ácido linoleico sobre a disfunção contrátil e bioenergética mitocondrial após infarto agudo do miocárdio. Tese ((Doutorado em Ciências Fisiológicas), Universidade Federal do Espírito Santo, 2017.

Santos J., Meira K. C., Camacho A.R., Salvador P. T. C. O., Guimarães R. M., Pierin A. M. G., Simões T. C., \& Freire F. H. M. A. (2018). Mortalidade por infarto agudo do miocárdio no Brasil e suas regiões geográficas: análise do efeito da idade-período-coorte. Rev Ciências e Saúde Coletiva. 25(5), 1621-1634.

Santos R. D., Gagliardi A. C. M., Xavier H. T., Magnoni C. D., Cassani R., Lottenberg A. M. P., Casella A. F., Araújo D. B., Cesena F. Y., Alves R. J., Fenelon G., Nishioka S. A. D., Faludi A.A., Geloneze B., Scherr C., Kovacs C., Tomazzela C., Carla C., Barrera-Arellano D., Cintra D., Quintão E., Nakandakare E. R., Fonseca F. A. H., Pimentel I., Santos J. E., Bertolami M.C., Rogero M., Izar M. C., Nakasato M., Damasceno N. R. T., Maranhão R., Cassani R. S. L., Perim R., \& Ramos S. (2013). I Diretriz sobre o consumo de gorduras e saúde cardiovascular. Arq. Bras. Cardiol., 100.

Takahashi M., Ando J., Shimada K., Nishizaki Y., Shigamasa T., Takayuki O., Yamamoto M., Nagao K., Hirayama A., Yoshimura M., Daida H., Nagai R., \& Komuro I. (2017). The ratio of serum n-3 to n-6 polyunsaturated fatty acids is associated with diabetes mellitus in patients with prior myocardial infarction: a multicenter cross-sectional study. BMC Cardiovasc Disord. 17(1), 41. 